\title{
REGALS: data analysis at the SAS frontier
}

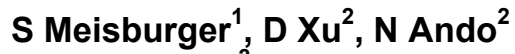 \\ ${ }^{1}$ Cornell University, Ithaca, ${ }^{2}$ Cornell University, Ithaca, NY \\ spm82@cornell.edu
}

SAS experiments at the frontier often seek to measure macromolecular components whose concentrations vary, as in ligand titrations, time-resolved methods, or chromatographic separations. These experiments produce large datasets that must be processed to extract the individual component scattering curves. Mathematically, the problem is related to matrix factorization and can be solved by singular value decomposition (SVD). However, the basis vectors from SVD are usually non-physical, and there is the additional problem of finding linear combinations that satisfy physically motivated restraints. Previously, we used evolving factor analysis (EFA) to analyze sizeexclusion chromatography (SEC) SAXS experiments with overlapping peaks [1]. The method was integrated into a well-known software package [2] and has been used by many research groups. However, EFA cannot handle severe peak overlap or changing background. To address a wider class of problems, we developed a computational method called REGALS (for REGularized Alternating Least Squares) that performs matrix factorization such that "physically-meaningful" solutions are enforced by regularization. Regularization functions chosen by the user are applied separately to each component and can include smoothness and extent of the concentration peak, smoothness of the SAXS profile, or smoothness and maximum dimension of the real-space P(r) function. We recently applied REGALS to ion-exchange (IEX) SAXS data, which cannot be analyzed by EFA because the salt gradient produces a changing background [3]. In my presentation I will describe the further development of REGALS and its performance on challenging chromatography datasets. In addition, I will explore the method's potential for other types of experiments, including equilibrium titrations and time-resolved SAXS. Finally, I will describe the REGALS software package, which is free and open-source and includes both Python and MATLAB implementations. References: [1] Meisburger SP, Taylor AB, Khan CA, Zhang S, Fitzpatrick PF, Ando N. (2016). Domain movements upon activation of phenylalanine hydroxylase characterized by crystallography and chromatography-coupled small-angle X-ray scattering. JACS 138(20): 6506-16. [2] Hopkins, J. B., Gillilan, R. E., \& Skou, S. (2017). BioXTAS RAW: improvements to a free open-source program for small-angle X-ray scattering data reduction and analysis. J. Appl. Crystallogr. 50(5): 1545-53. [3] Parker MJ, Maggiolo AO, Thomas WC, Kim A, Meisburger SP, Ando N, Boal AK, Stubbe J. (2018). An endogenous dAMP ligand in Bacillus subtilis class Ib RNR promotes assembly of a noncanonical dimer for regulation by dATP. PNAS 115(20): E4594-E4603.

Acta Cryst. (2020). A76, a145 